\title{
Effectiveness of gelatin solidification for unfrozen preservation and transportation of astrocytes
}

\author{
Nobuko Ishibashi $^{1}$, Shinichi Sotome ${ }^{1}$, Kazuyuki Nakajima ${ }^{2}$, Akio Shimizu $^{1 *}$ \\ ${ }^{1}$ Department of Environmental Engineering for Symbiosis, Faculty of Engineering, Soka University, Tokyo, Japan \\ ${ }^{2}$ Department of Bioinformatics, Faculty of Engineering, Soka University, Tokyo, Japan \\ Email: ${ }^{*}$ shimizu@soka.ac.jp
}

Received 17 June 2012; revised 19 July 2012; accepted 24 July 2012

\begin{abstract}
We herein studied a non-cryogenic cell preservation and transportation method. We found that astrocytes can be preserved in an unfrozen state at $4^{\circ} \mathrm{C}$ (without medium exchange), while maintaining good condition in comparison with cryopreservation for the periods of 5 - 7 days. The gelatin solidification method can also prevent decreases in survival rate by preventing damage to astrocytes during transportation. Therefore, the gelatin solidification method at $4^{\circ} \mathrm{C}$ may be a useful choice for short-term preservation and transportation.
\end{abstract}

Keywords: Cell Preservation; Gelatin; Astrocytes; Unfrozen Preservation; Transportation

\section{INTRODUCTION}

Cryopreservation at or near the temperature of liquid nitrogen, is commonly used in biological research. However, freezing induces cell death due to intracellular ice formation [1-5] or chemoosmotic stress [3,6-10], which results in plasma membrane disruption and subsequent necrosis. To prevent ice formation, numerous investigators have cryopreserved cellular systems using cryoprotective agents (i.e., dimethyl sulfoxide, glycerol or sugar) in an extracellular-like carrier solution, such as standard cell culture medium, but this approach is associated with harmful effects due to the cryoprotective reagents themselves $[11,12]$. Therefore, it is desirable that cells be preserved in an unfrozen state.

When cells are transported in culture medium in an unfrozen state, they are subjected to physical stress and damage. Therefore, cell solidification is important for transportation in such a state. Agar is widely used for cultivation and is considered to be harmless to cells; however, the melting temperature of agar is above $60^{\circ} \mathrm{C}$, and cells can be damaged at this temperature. Thus, we

"Corresponding author. attempted to use gelatin, which solidifies at about $15^{\circ} \mathrm{C}$ $20^{\circ} \mathrm{C}$. Gelatin is known to be safe for cells, as gelatincoated dishes are routinely used for cell culture, and gelatin was used as a solid medium before agar $[13,14]$.

For this experiment, we selected astrocytes, which are a sub-type of the central nervous glial cells, and are the most abundant cell in the human brain. They perform numerous functions, including biochemical support of endothelial cells that form the blood-brain barrier, provision of nutrients to the nervous tissue, maintenance of extracellular ion balance, and acting in the repair and scarring process of the brain and spinal cord following traumatic injuries $[15,16]$.

We studied the survival rate of these cells in an unfrozen state for up to one week, which would be necessary for domestic transport in Japan. We first compared the survival rate and metabolic activity of astrocytes between unfrozen preservation and cryopresevation. We then determined the optimal conditions (gelatin concentration, dissolution temperature, solidification temperature, etc.) for the gelatin solution, and investigated the influence of gelatin solidification on astrocyte survival. Finally, from the standpoint of transportation, the influence of physical stress on survival rates of astrocytes preserved under two different conditions (gelatin solidification and in solution) was investigated. Based on these results, we discuss the effects of gelatin solidification on survivability and protection from damage during transportation of astrocytes in an unfrozen state.

\section{MATERIAL AND METHODS}

\subsection{Materials}

Astrocytes were obtained from a neonatal rat brain-derived primary culture (CLEA Japan, Inc.). Trypsin and calf serum were obtained from GIBCO BRL. Dulbecco's modified eagle's medium (DMEM) and phosphate buffered saline (PBS) were purchased from Invitrogen. Gelatin from porcine skin type A and trypan blue stain were obtained from Sigma-Aldrich and Gibco, respectively. The CellTiter 
$96^{\circledR}$ AQueousOne Solution Cell Proliferation (MTS) assay kit and sodium dihydrogen phosphate dehydrate were purchased from Promega KK and Wako Pure Chemical Industries Ltd., respectively.

\subsection{Sample Preparation}

Astrocytes in DMEM containing 10\% FBS (fetal calf serum; GIBCO BRL) were incubated in a $\mathrm{CO}_{2}$ incubator for 20 - 30 days at $37^{\circ} \mathrm{C}$ under $5 \% \mathrm{CO}_{2} / 95 \%$ air. Viable astrocytes adhering to the surface of the culture flask were detached with $0.05 \%$ trypsin (GIBCO BRL). Detached astrocytes were centrifuged at $800 \mathrm{rpm}$ for $7 \mathrm{~min}$, and were suspended in DMEM. Suspended astrocytes were subjected to filtration (100- $\mu \mathrm{m}$ mesh), and the number of astrocytes was counted under a microscope.

The number of astrocytes used in this experiment was controlled to $1 \times 10^{6}$ in $300 \mu \mathrm{l}$ DMEM. This $300-\mu 1$ cell suspension was then placed into a $1.5-\mathrm{ml}$ microcentrifuge tube and preserved for 4 days at $4{ }^{\circ} \mathrm{C}$.

$10 \%$ gelatin stock solution was prepared by dissolving gelatin in PBS, and it was autoclaved $20 \mathrm{~min}$ at $120^{\circ} \mathrm{C}$. Gelatin solutions of various concentrations were prepared by dilution in DMEM at $35^{\circ} \mathrm{C}$. In this experiment, "in solution" refers to the experiment in which DMEM medium contained no gelatin. All of the added gelatin was dissolved in DMEM medium; thus, $1.5 \%$ gelatin refers to DMEM medium containing $1.5 \%$ gelatin.

\subsection{Astrocyte Preservation}

Astrocytes in solidified gelatin and in solution were preserved in a static state and under agitation for 4 days at $4^{\circ} \mathrm{C}$. To simulate transportation, cells were agitated at 20 $\mathrm{rpm}$ during the preservation period using a rotator (RT30mini; TAITEC) at $4^{\circ} \mathrm{C}$. As shown in previous section the number of astrocytes was controlled to $1 \times 10^{6}$ in 300 $\mu \mathrm{l}$ DMEM, and then preserved in a $1.5-\mathrm{ml}$ microcentrifuge tube.

Cryopreserved astrocytes were prepared by adding $5 \times$ $10^{5}$ astrocytes to $1.0 \mathrm{~mL}$ of preservation solution (Cell Banker). The cell suspension in a cryotube was placed on ice for $30 \mathrm{~min}$, followed by cooling to $-80^{\circ} \mathrm{C}$ and preservation for 2, 3, 4, 7 or 14 days. Cryopreserved astrocytes were quickly thawed in warm water at $35^{\circ} \mathrm{C}$ before use.

\subsection{Determination of Survival Rates and Metabolism}

After preservation under each condition, the number of viable cells was determined using the trypan blue staining method, which indicates membrane integrity; when the cell membrane is damaged, the dye penetrates through the membrane and dead cells are stained blue. The metabolic activity of cells was determined by MTS assay, a colorimetric assay for measuring the activity of enzymes that reduce MTS to formazan dyes, which have a purple color. After 4 days of preservation, each sample was incubated at $35^{\circ} \mathrm{C}$ in a constant-temperature water bath for $15 \mathrm{~min}$ in order to melt the gelatin (samples with no gelatin added were also incubated for comparison under the same conditions). In viable cells, MTS, which has a maximum absorbance at $490 \mathrm{~nm}$, is metabolized to formazan by succinate-tetrazolium reductase, leading to a decrease in absorbance at $490 \mathrm{~nm}$. From this change in absorbance, metabolic activity was determined.

\subsection{Morphological Observation}

As astrocytes are adherent cells, viable astrocytes adhere to culture plates and develop an increasing number of projections. In contrast, dead astrocytes do not adhere to culture plates and are suspended (spherical form) in medium. Therefore, the life and death of astrocytes can be qualitatively determined based on their shape in culture.

\section{RESULTS}

\subsection{Optimal Preservation Temperature for Astrocytes in Closed Vessel}

The optimal temperature for astrocyte survival in a closed vessel was determined, because during transport, we are unable to use a $\mathrm{CO}_{2}$ incubator or exchange medium. We performed experiments at three temperatures; $4^{\circ} \mathrm{C}$ (refrigeration temperature), $25^{\circ} \mathrm{C}$ (room temperature) and $37^{\circ} \mathrm{C}$ (incubation temperature). Figure 1 shows the survival rates and metabolic activities of astrocytes after 4 days of preservation. Significant viability was only observed at $4^{\circ} \mathrm{C}$, while the metabolic activity at $4^{\circ} \mathrm{C}$ was similar to that before preservation. Furthermore, astrocytes preserved at $4^{\circ} \mathrm{C}$ for 4 days adhered to the culture plate and showed an increasing number of projections (data not shown). These results confirm that $4^{\circ} \mathrm{C}$ is adequate for astrocyte preservation without medium exchange for 4 days or more. All subsequent unfrozen preservation experiments were thus performed at $4^{\circ} \mathrm{C}$.

\subsection{Influence of Freezing on Survival Rate and Metabolic Activity of Astrocytes}

Survival rates of astrocytes after cryopreservation and unfrozen preservation are shown in Figure 2.

Cryo-preserved astrocytes at $-80^{\circ} \mathrm{C}$ showed survival rates of approximately $80 \%$ up to 14 days. On the other hand, the survival rate of astrocytes preserved at $4^{\circ} \mathrm{C}$ decreased gradually with increasing preservation period. Therefore, cryopreservation becomes advantageous with longer preservation periods, as judged by the trypan blue method. In contrast, the metabolic activity of cryo-preserved astrocytes, as determined by MTS assay, decreased to 
about $50 \%$ at day 14 , regardless of preservation period (Figure 3), while that of astrocytes preserved at $4^{\circ} \mathrm{C}$ was over $70 \%$ up to day 7 (Figure 3).

\subsection{PH Changes of DMEM Medium after Addition of Gelatin and Influence on Survival Rate of Astrocytes}

The $\mathrm{pH}$ of DMEM medium decreased from 8.3 to 7.0 by addition of gelatin from $0 \%$ to $5 \%$ at room temperature (gelatin is not solidified at this temperature). Therefore, we measured the $\mathrm{pH}$ dependence of astrocyte survival rate in DMEM medium. The $\mathrm{pH}(6.8 \sim 8.4)$ was adjusted

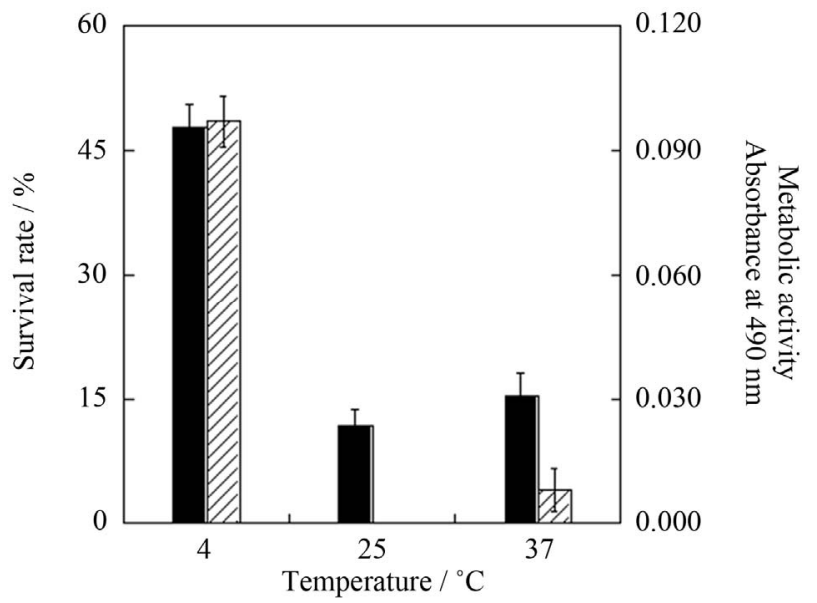

Figure 1. Survival rate (solid bars) and metabolic activity (hatched bars) of astrocytes after 4 days preservation at $4^{\circ} \mathrm{C}$, $25^{\circ} \mathrm{C}$ or $37^{\circ} \mathrm{C}$. Metabolic activity is shown in terms of absorbance at $490 \mathrm{~nm}$ on MTS assay. Mean $\pm \operatorname{SEM}(\mathrm{n}=3)$.

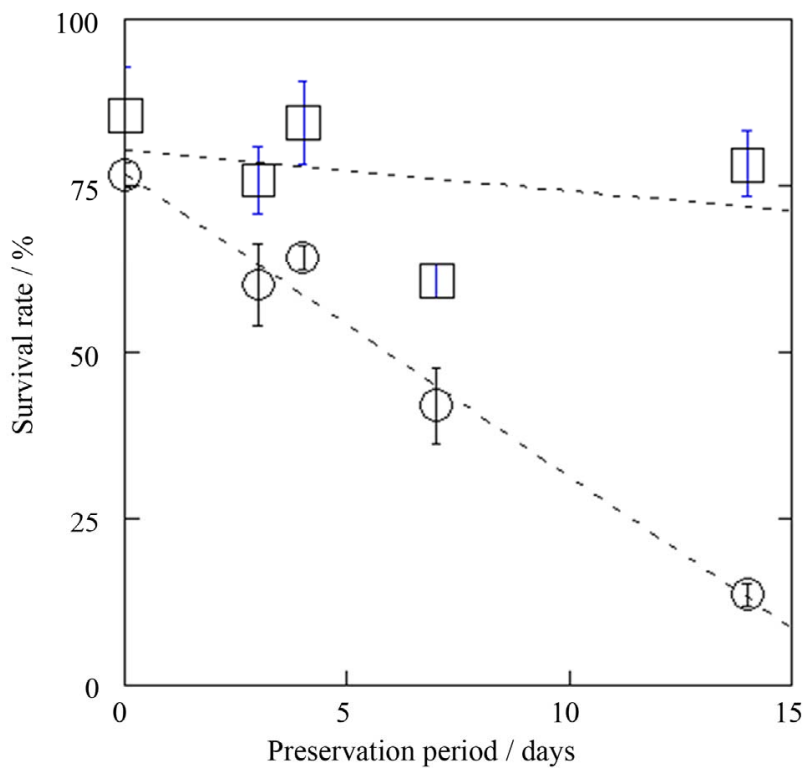

Figure 2. Preservation period dependence of survival rates for unfrozen $\left(4^{\circ} \mathrm{C}, \circ\right)$ and cryopreserved $\left(-80^{\circ} \mathrm{C}, \square\right)$ astrocytes. Mean $\pm \operatorname{SEM}(\mathrm{n}=3)$. with $\mathrm{H}_{2} \mathrm{NaPO}_{4}$ buffer, and the survival rate of astrocytes showed little change. Thus, we ignored the influence of $\mathrm{pH}$ changes on survival rate under in the present experiment condition, and used gelatin-DMEM solution without adjusting $\mathrm{pH}$.

\subsection{Adequate Gelatin Concentration for Astrocyte Solidification}

In order to solidify the cells in an adequate period at $4^{\circ} \mathrm{C}$, optimal gelatin conditions were determined. First, we measured the sedimentation time of astrocytes in $1.5-\mathrm{ml}$ microcentrifuge tubes at four gelatin concentrations $(0 \%$, $0.5 \%, 1.5 \%, 3.0 \%$ and $5.0 \%$ ) at $25^{\circ} \mathrm{C}$. The sedimentation time was $25 \mathrm{~min}$ at $1.5 \%$ and $3.0 \%$ gelatin concentrations at $25^{\circ} \mathrm{C}$, and these gelatin solutions were solidified with sufficient hardness within $15 \mathrm{~min}$ at $4^{\circ} \mathrm{C}$. However, the sedimentation time of astrocytes in $5.0 \%$ gelatin solution at $25^{\circ} \mathrm{C}$ was over $60 \mathrm{~min}$, and the solidification time in $0.5 \%$ gelatin solution at $4^{\circ} \mathrm{C}$ was more than $300 \mathrm{~min}$. Therefore, $1.5 \%$ and $3.0 \%$ gelatin solutions were used in subsequent experiments.

\subsection{Influence of Gelatin Concentration on Survival Rate of Astrocytes in Standing Preservation}

Survival rates of astrocytes solidified at various gelatin concentrations $(0 \%, 1.5 \%$ and $3.0 \%)$ were determined after standing preservation for 4 days at $4^{\circ} \mathrm{C}$ in order to clarify the effects of gelatin solidification. For astrocytes preserved in solution and those solidified in $1.5 \%$ gelatin, the survival rate was about $65 \%$, however, the survival rate of astrocytes in $3 \%$ gelatin was about $10 \%$ lower.

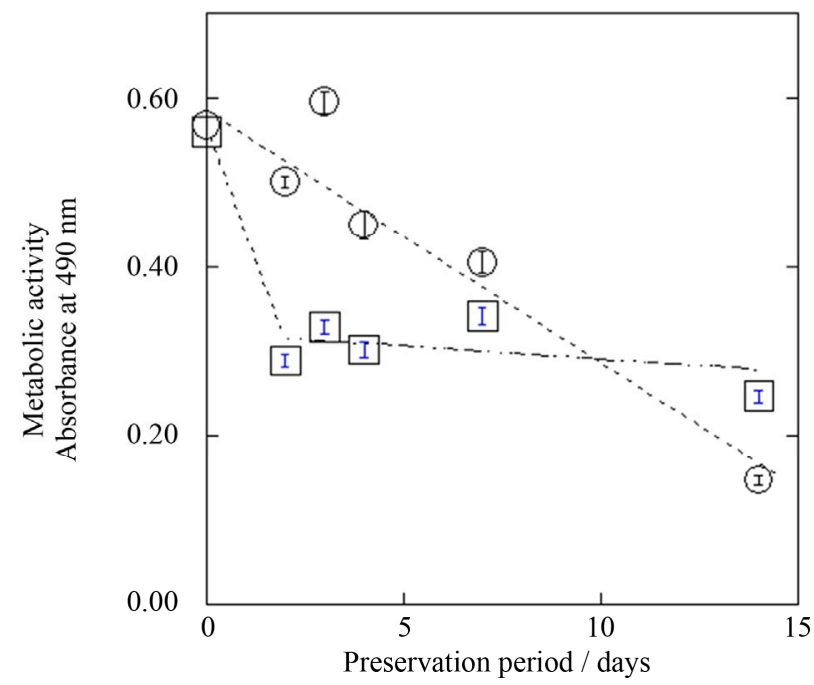

Figure 3. Preservation period dependence of metabolic activities of unfrozen $\left(4^{\circ} \mathrm{C}, \circ\right)$ and cryopreserved $\left(-80^{\circ} \mathrm{C}, \square\right)$ astrocytes. Metabolic activity is shown in terms of absorbance at $490 \mathrm{~nm}$ on MTS assay. Mean \pm SEM $(n=3)$. 
Based on the results for sedimentation time, solidification time and influence on survival rate, we studied the effects of physical impact during transportation on survival rates of astrocytes in solution (DMEM medium) and solidified in $1.5 \%$ gelatin-DEME medium.

\subsection{Influence of Physical Impact (Agitation by Rollover Stirring) on Survival Rates of Astrocytes Preserved in Solidified Gelatin and Those in Solution}

The effects of physical impact (agitation by rollover stirring) on the survival rate of astrocytes preserved in solidified gelatin was compared with that of astrocytes preserved in solution at $4^{\circ} \mathrm{C}$ for 4 days (Figure 4).

After 4 days, the survival rate of astrocytes (5\%) subjected to physical impact was about 50\% lower when compared to those preserved in standing solution (54\%). On the other hand, in astrocytes preserved in solidified with gelatin, the survival rate was unchanged. In solution, physical impact dispersed and disrupted astrocytes in the preservation medium, and morphology was affected in about $75 \%$ of cells. However, astrocytes solidified in gelatin were fixed at the bottom of vessel and were not injured at all.

\subsection{Influence of Physical Impact on Metabolic and Growth Activity of Astrocytes}

The absorbance (MTS assay) under two conditions (solidified in $1.5 \%$ gelatin and dispersed in solution) after 4 days of standing preservation at $4{ }^{\circ} \mathrm{C}$ was consistent. This indicates that there are no differences in the metabolic

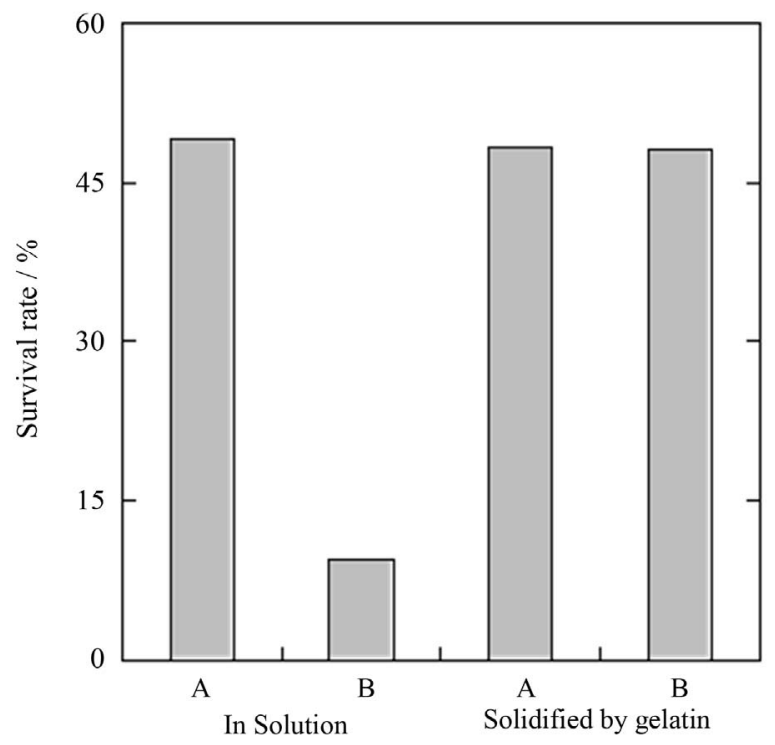

Figure 4. Survival rate of astrocytes after 4 days of preservation at $4{ }^{\circ} \mathrm{C}$ in solution and solidified in gelatin. A: Preserved under static conditions; B: Preserved with agitation by rollover stirring. activity of viable astrocytes under these preservation conditions. Growth activity also showed similar values, irrespective of preservation method (data not shown). Therefore, the metabolic and growth activity for viable astrocyte after preservation by various methods showed no significant differences.

\section{DISCUSSION}

The survival rate of astrocytes in a standing state without medium exchange at $4^{\circ} \mathrm{C}$ was clearly higher than at $25^{\circ} \mathrm{C}$ and $37^{\circ} \mathrm{C}$. The survival rate of cryopreserved astrocytes was higher than after unfrozen preservation at $4^{\circ} \mathrm{C}$ for preservation periods of up to one week. Clear differences were observed after 5 or 6 days of preservation. However, the metabolic activity of cryopreserved astrocytes was lower than that of astrocytes preserved at $4^{\circ} \mathrm{C}$. This indicates that some of the cryopreserved astrocytes judged as viable cells had lost metabolic activity, or that the metabolic activity of all of viable astrocytes was decreased by freezing. Therefore, we compared the "metabolic activity ratio", which is defined as the survival rate multiplied by the metabolic activity. Here, metabolic activity (normalized against the same living cell number) is calculated by dividing the absorbance at $490 \mathrm{~nm}$ observed on MTS assay before preservation by that after preservation. Figure 5 shows the metabolic activity ratio of astrocytes determined by above method after preservation at $-80^{\circ} \mathrm{C}$ and at $4^{\circ} \mathrm{C}$, and it was revealed that astrocytes preserved at $4^{\circ} \mathrm{C}$ maintained good condition (high metabolic activity ratio) when compared with cryopreserved cells over periods of 4 or 5 days.

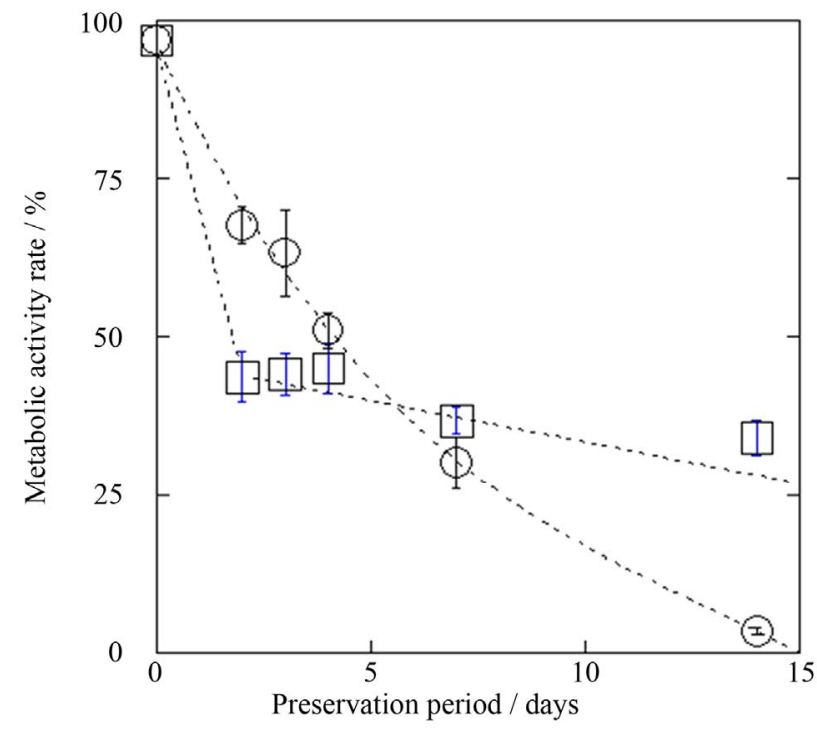

Figure 5. Preservation period dependence of metabolic activity rates of refrigerated $\left(4^{\circ} \mathrm{C}, \circ\right)$ and cryopreserved $\left(-80^{\circ} \mathrm{C}, \square\right)$ astrocytes. Here, metabolic activity is normalized against survival rate, which was determined by absorbance at $490 \mathrm{~nm}$ on MTS assay. Mean $\pm \operatorname{SEM}(\mathrm{n}=3)$. 
On the other hand, when dispersed in solution, cells are disrupted due to movement during transport. Thus, we solidified the astrocytes in gelatin. Both the melting temperature and solidification time of $1.5 \%$ gelatin were adequate for astrocyte preservation, because $1.5 \%$ gelatin solution solidified in approximately $15 \mathrm{~min}$ at $4^{\circ} \mathrm{C}$ and melted in approximately $5 \mathrm{~min}$ at about $37^{\circ} \mathrm{C}$. When astrocytes were preserved for 4 days in solidified gelatin, the survival rate after physical impact was the same as those preserved in the standing state. In contrast, when astrocytes were preserved in solution, the survival rate in the standing state was $54 \%$, and this decreased to about $5 \%$ after physical impact. Similar results were seen for metabolic activity.

Gelatin solidification has no risk of harmful effects, in contrast to cryoprotective agents, and there is no risk of injuries due to ice crystals $[1-5,11,12]$. In fact, metabolic activity rate was lower with cryopreservation $\left(-80^{\circ} \mathrm{C}\right)$, which is a typical preservation method for periods of up to about five days, as compared with the gelatin solidification method at $4^{\circ} \mathrm{C}$. These results confirm that astrocytes can be preserved in an unfrozen state at $4^{\circ} \mathrm{C}$ (without medium exchange) while maintaining good condition in comparison with cryopreservation for periods of 5 - 7 days. In addition, gelatin solidification is inexpensive, simple and effective for preventing damage to astrocytes during transportation. Therefore, gelatin solidification at $4^{\circ} \mathrm{C}$ is a useful option for short-term cell preservation and transportation.

\section{ACKNOWLEDGEMENTS}

This research was supported by the MEXT Program for Strategic Research Foundation at Private Universities, 2010-2014.

\section{REFERENCES}

[1] Acker, J.P., Elliott, J.A.W. and McGann, L.E. (2001) Intercellular ice propagation: Experimental evidence for ice growth through membrane pores. Biophyicals Journal, 81, 1389-1397. doi:10.1016/S0006-3495(01)75794-3

[2] Acker, J.P. and McGann, L.E. (2002) Innocuous intracellular ice improves the survival of frozen cells. Cell Transplant, 11, 563-571.

[3] Mazur, P. (1984) Freezing of living cells: Mechanisms and implications. American Journal of Physiology, 247, C125-C147.
[4] David, P.E. (1976) Long-term preservation of cells and tissues: A review. Journal of Clinical Pathology, 29, 271285. doi:10.1136/jep.29.4.271

[5] Acker, J.P. and McGann, L.E. (2003) Protective effect of intracellular ice during freezing? Cryobiology, 46, 197202. doi:10.1016/S0011-2240(03)00025-7

[6] Hubálek, Z. (2003) Protectants used in the cryopreservation of microorganisms. Cryobiology, 46, 205-229. doi:10.1016/S0011-2240(03)00046-4

[7] Lozina-Lozinsk, L.K. (1965) Survival of some insects and cells following intracellular ice formation. Federation Proceedings, 15, S206-S211.

[8] McGann, L.E. (1978) Differing actions of penetrating and nonpenetrating cryoprotective agents. Cryobiology, 15, 382-390. doi:10.1016/0011-2240(78)90056-1

[9] Odintsova, N., Kiselev. K., Sanina, N. and Kostetsky, E. (2001) Cryopreservation of primary cell cultures of marine invertebrates. CryoLetters, 22, 299-310.

[10] Baust, J.M., Buskirk, R.V. and Baust, J.G. (2000) Cell viability improves following inhibition of cryopreservation-induced apoptosis. In Vitro Cellular \& Developmental Biology-Animal, 36, 262-270. doi:10.1290/1071-2690(2000)036<0262:CVIFIO >2.0.CO :2

[11] Paynter, S., Cooper, A., Thomas, N. and Fuller, B. (1996) Cryopreservation of multicellular embryos and tissues. In: Karow, A. and Critser, J., Eds., Reproductive Tissue Banking, Academic Press, New York, 1997.

[12] Polge, C., Smith, A.U. and Parkes, A.S. (1949) Revival of spermatozoa after vitrification and dehydration at low temperatures. Nature, 164, 666. doi:10.1038/164666a0

[13] Frankland, J.C., Latter, P.M. and Poskitt, J.M. (1995) A laboratory guide to solid microbiology: Some general principles and practice. Medewood Research and Development Paper, 115, 1-79.

[14] Kim, N.S. and Kim, S.J. (1991) Isolation and cultivation of microvascular endothelial cells from rat lungs: Effects of gelatin substratum and serum. Yonsei Medical Journal, 32, 303-314.

[15] Araque, A., Parpura, V., Sanzgiri, R.P. and Haydon, P.G. (1999) Tripartite synapses: Glia, the unacknowledged partner. Trends in Neurosciences, 22, 208-215. doi:10.1016/S0166-2236(98)01349-6

[16] Kolb, B and Whishaw, I.Q. (2008) Fundamentals of human neuropsychology. 6th Edition, Worth, New York. 